Revista Colombiana de Obstetricia y Ginecología Vol. 55 No.2 • 2004 • (136-145)

Artículo de Revisión

\title{
¿CUÁNDO PINZAR EL CORDÓN UMBILICAL?
}

\section{WHIEN TO CLAMP THE UIMBILICAL CORD?}

Fernando Arango Gómez, M.D. ", Juan Carlos Mejía Londoño, M.D."*

Recibido: mayo 20/2004 - Revisado: junio 11/2004 - Aceptado: junio 25/2004

\section{RESUMEN}

El pinzamiento del cordón umbilical la mayoría de las veces se realiza en forma mecánica y casi inmediata al nacimiento, sin detenerse a pensar en las posibles implicaciones que esta conducta puede tener en el recién nacido o en la madre. En el presente artículo se revisa la evidencia disponible con respecto a los conceptos fisiológicos inherentes al pinzamiento del cordón, las razones por las que se ha recomendado el pinzamiento temprano, los posibles efectos benéficos o perjudiciales al momento del pinzamiento y las recomendaciones del Ministerio de la Protección Social.

Palabras clave: cordón umbilical, pinzamiento del cordón umbilical, adaptación neonatal.

\section{SUIMMARY}

The umbilical cord is usually clamped mechanically and immediately after birth, without thinking about the implications in the newborn or the mother. In this review we present the available evidence with regard to the physiologic concepts about cord clamping, why is immediately clamping a common practice, the potential adverse effects of early clamping, the potential beneficial effects of delayed clamping, and the Ministerio de la Protección Social (Ministry for Social Protection) recommendations.

Key words: umbilical cord, umbilical cord clamping, neonatal adaptation.

\footnotetext{
Pediatra Neonatólogo, Profesor Auxiliar, Universidad de Caldas, Manizales, Colombia.

** Residente de Pediatría, Universidad de Caldas.
}

\section{INTRODUCCIÓN}

En muchas culturas las personas creen que en el momento del nacimiento se debe transferir la vida de la placenta hacia el bebé o de lo contrario éste podría morir, y el cordón umbilical se corta luego de que cesen las pulsaciones o se desprenda la placenta. En otras áreas, el cordón es “ordeñado”, especialmente si el bebé nace deprimido, con el fin de devolverle su alma. ${ }^{1}$ En la mayoría de los hospitales occidentales, el pinzamiento del cordón umbilical se realiza rutinariamente en forma inmediata o temprana, sin pensar en las posibles implicaciones para el recién nacido o la madre. En una encuesta realizada hace más de 50 años, ${ }^{2}$ la mayoría de los médicos creía que el momento del pinzamiento del cordón umbilical no era importante. Desde entonces, no se han publicado otras encuestas de opinión de los médicos al respecto, pero sí de las enfermeras obstetrices de Estados Unidos, ${ }^{3}$ en la que se reflejan las diferentes prácticas y creencias con respecto al tema.

A pesar de muchos años de discusión, debate y diálogo, existe poco acuerdo acerca del momento óptimo para pinzar el cordón umbilical después del nacimiento. De igual forma, no hay consenso ni evidencia científica suficiente acerca de los posibles efectos benéficos o perjudiciales para el recién nacido, que puedan atribuirse como consecuencia del pinzamiento temprano o tardío. ${ }^{4}$ Prueba de ello son las diferentes recomendaciones dadas por los autores de los textos clásicos de obstetricia ${ }^{5-8}$ (tabla 1) y por las sociedades científicas. Por ejemplo, el Colegio Americano de Obstetras y Ginecólogos en conjunto con la Academia Americana de Pediatría 


\begin{tabular}{|c|c|c|c|}
\hline Texto & Pinzamiento & Posición del bebé & Referencia \\
\hline $\begin{array}{l}\text { Obstetricia de } \\
\text { William's }\end{array}$ & A los 30 segundos. & $\begin{array}{l}\text { No elevarlo por encima del } \\
\text { introito vaginal. }\end{array}$ & 5 \\
\hline $\begin{array}{l}\text { Obstetricia de } \\
\text { Schwartcz }\end{array}$ & $\begin{array}{l}\text { Cese de los latidos. Ligadura inmediata } \\
\text { si hay depresión neonatal o la madre es } \\
\text { Rh negativa sensibilizada. }\end{array}$ & Plano inferior al del útero. & 6 \\
\hline $\begin{array}{l}\text { Obstetricia y Gine- } \\
\text { cología. Texto } \\
\text { integrado }\end{array}$ & $\begin{array}{l}\text { Cese de los latidos; ligadura inmediata si } \\
\text { hay depresión neonatal o la madre es Rh } \\
\text { negativa sensibilizada. }\end{array}$ & No hace mención. & 7 \\
\hline Obstetricia de Beck & No hace mención. & No hace mención. & 8 \\
\hline
\end{tabular}

no mencionan este tema en sus Guías de Cuidado Perinatal; ${ }^{9}$ la Sociedad de Obstetras y Ginecólogos del Canadá recomienda pinzamiento inmediato del cordón umbilical para la obtención rutinaria de sangre del mismo para análisis de gases sanguíneos $;^{10}$ recomendación similar es dada por el Colegio Real de Obstetras y Ginecólogos del Reino Unido. ${ }^{11}$ Desconocemos la posición de la Sociedad Colombiana de Obstetricia y Ginecología ${ }^{12}$ y de la Sociedad Española de Ginecología y Obstetricia ${ }^{13}$ y en sus páginas web no se publican sus políticas.

\section{CONCEPTOS FISIOLÓGICOS}

\section{Transfusión placentaria}

En condiciones naturales, una vez nace el bebé y mientras las arterias umbilicales se constriñen espontáneamente, la placenta le transfiere sangre oxigenada permitiendo dos hechos importantes, mantener la respiración placentaria y aumentar el volumen sanguíneo. La vena umbilical tensa se observa con cada contracción uterina, lo que indica que está pasando sangre fetal de la placenta hacia el recién nacido. Finalmente, la vena umbilical se constriñe, por lo general después de que el niño está rosado. Habitualmente, este proceso tarda tres minutos. ${ }^{14}$

Demorar el pinzamiento del cordón umbilical hasta que deje de pulsar permite el tiempo suficiente para que ocurra la transfusión placentaria, que le aporta al recién nacido cerca de un 30\% adicional de volumen sanguíneo y hasta un 60\% más de eritrocitos $^{15}$ (gráfica 1), los cuales al destruirse por hemólisis suplen alrededor de $50 \mathrm{mg}$ de hierro a las reservas del niño. ${ }^{16}$

La posición en que se mantiene al bebé influye en la transfusión placentaria. Se ha demostrado que retardar el pinzamiento durante 45 segundos, manteniendo al recién nacido a nivel del introito vaginal resulta en un incremento del $11 \%$ en el volumen sanguíneo y del 24\% de los glóbulos rojos. Mantenerlo a $30 \mathrm{~cm}$ por encima del introito retarda la transfusión placentaria y colocarlo a $30 \mathrm{~cm}$ por debajo acelera la transfusión placentaria de tres a un minuto. ${ }^{4,17}$

\section{Pinzamiento del cordón umbilical y adaptación neonatal}

Tradicionalmente, los cambios respiratorios y hemodinámicos que ocurren durante la transición de la vida intrauterina a la extrauterina se explican inicialmente por una adecuada expansión pulmonar y el incremento subsecuente del $\mathrm{pH}$ y la $\mathrm{PaO}_{2}$; se produce vasodilatación de la arteria pulmonar, disminución de la resistencia vascular y aumento del flujo sanguíneo a este órgano. En consecuencia, según este concepto, para que haya una adecuada perfusión pulmonar deben insuflarse primero los alvéolos. ${ }^{18}$ 


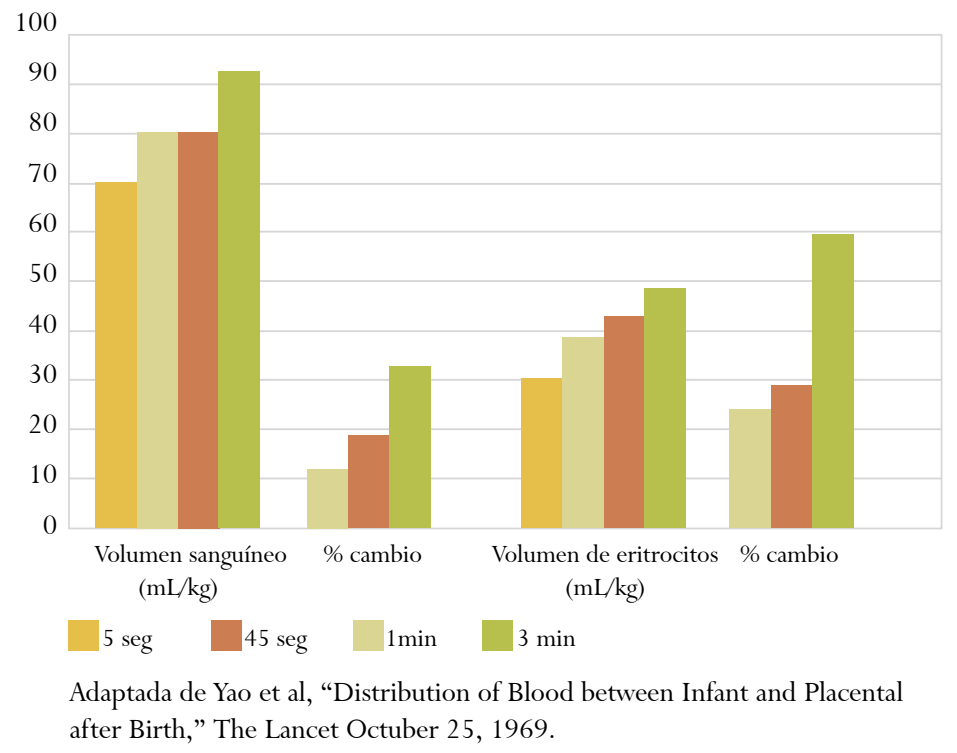

Gráfica 1. Cambios en el volumen sanguineo y eritrocitario según el momento de pinzamiento del cordón umbilical.

Recientemente se propuso un nuevo modelo de la fisiología de la adaptación neonatal. En la vida intrauterina los pulmones reciben únicamente el $8 \%$ del gasto cardiaco mientras que la placenta el $40 \%$. En el segundo periodo del trabajo de parto, las contracciones uterinas crean presiones mayores de $80 \mathrm{~mm} \mathrm{Hg}$, permiten el paso adicional de sangre de la placenta al feto inmediatamente antes o durante el nacimiento, mejoran la perfusión pulmonar y de otros órganos, llevan a la erección de los capilares pulmonares, incrementan el gasto cardiaco que va a los pulmones, facilitan la remoción del líquido pulmonar fetal y la entrada de aire a los alvéolos. La circulación umbilical continúa aportando oxígeno, que a la vez expande el volumen sanguíneo y corrige el pH. El incremento en los niveles de oxígeno estimula el centro respiratorio en el cerebro y comienza la respiración continua. El aumento en la $\mathrm{PaO}_{2}$ conduce al cierre de las arterias umbilicales; posteriormente ocurre estasis en la vena umbilical y se desprende la placenta. ${ }^{19}$ Según este nuevo modelo, para que haya una adecuada ventilación pulmonar deben perfundirse en forma adecuada los pulmones.

El concepto de erección capilar fue demostrado por Jaykka, quien en 1957 diseñó un experimento para evaluar el proceso de insuflación pulmonar, para lo cual utilizó pulmones de mortinatos humanos y de fetos de corderos. Evaluó el efecto de la insuflación sola, el efecto de infundir tinta a través de la arteria pulmonar para simular la perfusión pulmonar, y una combinación de los dos métodos. Inicialmente insufló los pulmones sólo con aire y encontró que la expansión no ocurría en forma uniforme. Tuvo dificultades al inyectar la tinta para simular la circulación capilar cuando intentó hacerlo luego de la insuflación. $\mathrm{Al}$ examen microscópico, las paredes alveolares eran irregulares y delgadas alrededor de los espacios aéreos globulares con áreas considerables que permanecían sin teñirse, representando acinos alveolares no reclutados (gráfica 2-A). Posteriormente, en otros pulmones, inyectó tinta en la arteria pulmonar con una presión de $80 \mathrm{~mm} \mathrm{Hgy}$ encontró que el sistema capilar se tornaba rígido o erecto, formando un marco que soportaba la unidad respiratoria. Microscópicamente, el cuadro se asemejaba al del pulmón normalmente aireado (gráfica 2-B). Por último, inyectó tinta en la arteria pulmonar de otros pulmones bajo presión y posteriormente los insufló. Requirió menos presión para insuflar los pulmones cuando el sistema vascular ya estaba distendido con la tinta. El cuadro microscópico se asemejó al de los pulmones normalmente aireados y fue similar al de los pulmones que habían sido tratados en forma experimental con líquido inyectado al sistema vascular bajo presión (gráfica 2-C). Concluyó que este proceso de erección capilar es un paso esencial en la adaptación cardiopulmonar neonatal. ${ }^{20,21}$ En una modificación de este experimento, la doctora Avery encontró también que era más fácil insuflar los pulmones si eran perfundidos previamente. ${ }^{22}$ Estos estudios soportan el concepto que el establecimiento de la respiración neonatal normal se basa en el flujo adecuado de sangre al lecho pulmonar. 


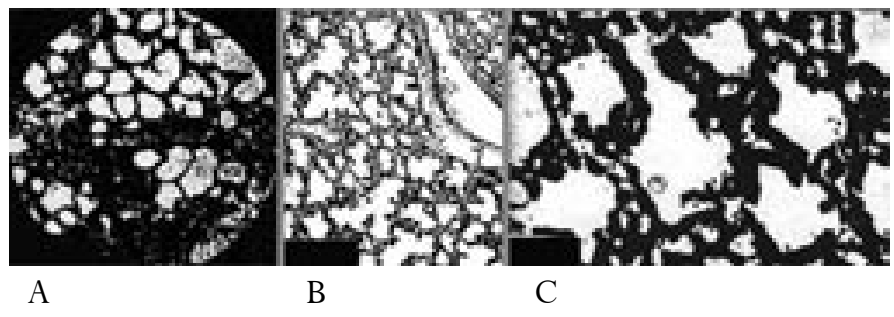

Gráfica 2. Erección capilar. Ver la descripción en el texto. Modificado de Jaykka S. An experimental study of the effect of liquid pressure applied to the capilary network of excised fetal lungs. Acta Paediatr 1957;Supl 112:2-91.

\section{¿POR QUÉ SE HA RECOMENDADO EL PINZAMIIENTO TEMPRANO?}

Hace algunas décadas se recomendaba esperar entre uno a cinco minutos después del nacimiento antes de pinzar el cordón umbilical. Las posibles razones por las que se abandonó esta práctica, especialmente en los hospitales del hemisferio occidental, son: ${ }^{23}$

1. Temor al desarrollo de policitemia, hiperviscosidad, hiperbilirrubinemia y taquipnea transitoria del recién nacido.

2. La presencia de un pediatra o un neonatólogo en la sala de partos "ansioso" por comenzar la atención del bebé.

3. El deseo de obtener sangre del cordón umbilical para medición de $\mathrm{pH}$ y gases como método de tamizaje de asfixia perinatal.

4. La necesidad percibida de iniciar el contacto piel a piel con la madre y la lactancia materna tan pronto como sea posible.

5. Para realizar manejo activo del alumbramiento y disminuir la hemorragia postparto.

A continuación se revisa la evidencia científica disponible con respecto al temor de aumentar ciertas patologías o condiciones neonatales.

\section{Policitemia}

La policitemia se define como un hematocrito venoso mayor de 65\% a 70\% y se ha relacionado con secuelas neurológicas. La asociación entre pinzamiento tardío del cordón y la policitemia se originó en un estudio descriptivo realizado en 1977 por Saigal y Usher, ${ }^{24}$ quienes describieron un subgrupo de recién nacidos que desarrollaron "plétora neonatal sintomática" con varios tiempos de pinzamiento del cordón umbilical. En 1992 se realizó un estudio clínico aleatorizado y no se encontraron diferencias en los resultados neurológicos a los 30 meses de seguimiento entre los niños con antecedente de policitemia neonatal comparados con aquellos sin policitemia. En la parte más extrema del protocolo, los recién nacidos a término y prematuros fueron mantenidos a $30 \mathrm{~cm}$ por debajo del introito vaginal $\mathrm{y}$ el pinzamiento del cordón se retardó durante cinco minutos. Ninguno de los prematuros desarrolló policitemia, pero dos de los recién nacidos a término tuvieron niveles de hematócrito mayores al 70\% a las cuatro horas de vida, uno de ellos se tornó sintomático. ${ }^{25}$ Este estudio es el único que sugiere una asociación entre el pinzamiento tardío del cordón umbilical y la policitemia, y sus resultados no han sido replicados.

Otras causas de policitemia mejor documentadas que el pinzamiento tardío del cordón son condiciones maternas pre-existentes, tales como diabetes, pre-eclampsia e hipertensión arterial, que aumentan el riesgo de hipoxia crónica intrauterina; la eritropoyesis resultante puede producir policitemia al nacer.

En una revisión sistemática de la literatura de los estudios clínicos aleatorizados y estudios clínicos controlados de las dos últimas décadas, se concluyó que no hay evidencia científica suficiente para afirmar que el pinzamiento tardío del cordón causa policitemia sintomática. ${ }^{4}$

\section{Hiperviscosidad}

Usualmente, pero no siempre, el aumento de la viscosidad sanguínea acompaña a la policitemia y se ha asociado con pobre resultado neurológico, aunque los estudios más recientes han fallado para documentar cualquier patrón de daño neurológico. Tanto las transfusiones sanguíneas como la transfusión placentaria fisiológica, cuando se pinza tardíamente el cordón umbilical, incrementan la viscosidad sanguínea en los recién nacidos. Dicho incremento 
se acompaña de una disminución significativa en la resistencia vascular que produce mayor vasodilatación pulmonar y sistémica, componentes esenciales de la adaptación neonatal a la vida extrauterina. ${ }^{4}$

\section{Hiperbilirrubinemia}

La preocupación se originó en 1972 con un reporte de niveles mayores de bilirrubinas en los recién nacidos prematuros, cuyos cordones umbilicales fueron pinzados en forma tardía. ${ }^{26}$ En la revisión sistemática descrita anteriormente,${ }^{4}$ se incluyeron cuatro estudios clínicos aleatorizados con 409 recién nacidos a término y siete con 247 recién nacidos prematuros; no se encontraron diferencias significativas en los niveles de bilirrubina en los niños con pinzamiento tardío comparados con aquellos con pinzamiento temprano.

\section{Taquipnea transitoria del recién nacido} Los defensores del pinzamiento temprano del cordón umbilical postulan que la policitemia y la hiperviscosidad resultantes del pinzamiento tardío incrementan la presión venosa central, con disminución del retorno venoso y linfático, compromiso de la reabsorción del líquido pulmonar fetal y aumento del riesgo de taquipnea transitoria del recién nacido. Sin embargo, los estudios clínicos controlados realizados en las últimas dos décadas no evidencian mayor incidencia de esta afección en los niños en quienes se realizó el pinzamiento tardío del cordón. En cambio sí hay reportes de mayor frecuencia respiratoria durante las primeras tres horas de vida que no requiere tratamiento. ${ }^{4}$

\section{¿EL PINZAMIENTO TARDÍO DEL CORDÓN PRODUCE BENEFICIOS?}

Aunque la mayoría de los estudios clínicos controlados incluyen grupos pequeños de pacientes y requieren ser replicados, los resultados sugieren varios efectos benéficos importantes.

\section{Beneficios hematológicos}

En los recién nacidos a término se han descrito niveles de hematócrito más altos a los dos y tres meses de edad y una tendencia a niveles mayores de ferritina sérica en el grupo de pacientes con pinzamiento tardío del cordón umbilical (tabla 2). ${ }^{27,28}$

En los recién nacidos prematuros se han demostrado niveles más altos de hemoglobina y hematócrito, y necesidades menores de transfusiones en las primeras seis semanas de vida. Antes del parto se aleatorizaron 40 recién nacidos menores de 33 semanas de edad gestacional a pinzamiento temprano (20 segundos) o tardío (45 segundos). Al finalizar la sexta semana, 16 pacientes en el grupo de pinzamiento temprano habían recibido transfusión de glóbulos rojos empacados, contra nueve en el grupo de pinzamiento tardío ( $p<0,05-$ OR 0,56 [0,34 $-0,94])$. En ningún paciente se encontraron signos de sobrecarga de volumen (hipertensión arterial, bradicardia persistente) o de viscosidad excesiva. La necesidad y duración de fototerapia fue similar en los dos grupos. Los autores concluyeron que retardar el pinzamiento del cordón umbilical por 45 segundos en los prematuros menores de 33 semanas de edad gestacional es posible, seguro y benéfico. $^{29}$

\begin{tabular}{|c|c|c|c|}
\hline \multicolumn{2}{|l|}{$\begin{array}{l}\text { Variable } \\
\text { (media) }\end{array}$} & \multirow{2}{*}{$\begin{array}{c}\begin{array}{c}\text { Pinzamiento } \\
\text { temprano }\end{array} \\
8,9\end{array}$} & \multirow{2}{*}{$\begin{array}{c}\begin{array}{c}\text { Pinzamiento } \\
\text { tardío }\end{array} \\
9,2\end{array}$} \\
\hline Hemoglobina & Materna & & \\
\hline & Cordón & 13,9 & 14,1 \\
\hline & 3 meses & 8,8 & 9,9 \\
\hline \multirow[t]{3}{*}{ Ferritina } & Materna & 25 & 26 \\
\hline & Cordón & 143 & 116 \\
\hline & 3 meses & 80 & 105 \\
\hline \multicolumn{2}{|c|}{ Cambio de $\mathrm{Hb}$ (0 a 3 meses) } & $-5,2$ & $-4,0$ \\
\hline
\end{tabular}

Adaptada de Geethanath RM, Ramji S, Thirupuram S, et al. Effect of timing of cord clamping on the iron status of infants at 3 months. Ind Pediatr 1997;34:103-106. 
En otro estudio realizado en Australia, 46 recién nacidos prematuros de 26 a 33 semanas de edad gestacional fueron asignados en forma aleatoria a pinzamiento inmediato o a los 30 segundos luego del nacimiento por vía vaginal o por cesárea. Se midió el hematócrito a la hora y cuatro horas de edad. Hubo tendencia a hematócritos más altos en los niños con pinzamiento más tardío, pero las diferencias no fueron significativas a la hora (55 \pm 7,7 vs. $52,9 \pm 7$ ) o a las cuatro horas de vida (55 \pm 7 vs. $52,5 \pm 7$ ). Los resultados sugieren que los estudios futuros deben realizarse retardando el pinzamiento por más de 30 segundos o alterando la posición del niño en relación con el útero a fin de facilitar la transfusión placentaria. Una conclusión importante de este estudio es que es posible pinzar tardíamente el cordón umbilical en los prematuros que nacen por cesárea. ${ }^{30}$

Finalmente, en un estudio reciente con 32 recién nacidos prematuros se encontró menor frecuencia de transfusiones sanguíneas en las primeras cuatro semanas de vida, menos transfusiones de albúmina en las primeras 24 horas, presión arterial media más alta en las primeras cuatro horas de vida e incrementos significativos en el hematócrito, hemoglobina y recuento de glóbulos rojos en los prematuros con pinzamiento tardío del cordón, comparados con los que se pinzaron en forma temprana. ${ }^{31}$

Aun con los tamaños de muestra pequeños en los estudios mencionados, tanto en recién nacidos a término como en prematuros, los hallazgos indican efectos benéficos hematológicos y soportan la necesidad de estudios futuros. ${ }^{4}$ Los efectos positivos del pinzamiento tardío del cordón umbilical podrían ser más importantes, tanto clínica como económicamente en los niños de los países en desarrollo, en quienes una transfusión placentaria moderada puede representar un medio fisiológico y económico de incrementar los depósitos de hierro. ${ }^{32}$

\section{Beneficios cardiopulmonares}

Los estudios sugieren que, tanto los recién nacidos a término como los prematuros tienen mejor vasodilatación pulmonar y sistémica, y mayor flujo sanguíneo al cerebro e intestino. ${ }^{33-35}$ En los recién nacidos de pretérmino se ha reportado incremento en la presión arterial ${ }^{31,33,36}$ y mejor adaptación cardiopulmonar con menor necesidad de oxígeno, y días de ventilación mecánica. ${ }^{4}$ En los recién nacidos a término, mejor llenado capilar, ${ }^{37}$ temperatura periférica más alta ${ }^{38}$ y mayor gasto urinario ${ }^{39}$ por la mayor perfusión debida al pinzamiento tardío del cordón umbilical.

\section{Otros beneficios potenciales}

El pinzamiento tardío del cordón representa un cambio en la rutina, que favorece el contacto temprano entre la madre y su hijo. Se ha demostrado una asociación estadísticamente significativa entre el contacto temprano y la duración de la lactancia materna, ${ }^{40}$ la cual fue más prolongada en los recién nacidos con pinzamiento tardío del cordón. ${ }^{41}$

\section{¿EXISTEN EFECTOS PERJUIDICIALES CON EL PINZAMIENTO TEMPRANO?}

En una revisión sistemática de la literatura referente al pinzamiento del cordón umbilical, no se encontraron efectos perjudiciales directos relacionados con el pinzamiento temprano en recién nacidos a término ni en prematuros, excepto un incremento de la anemia en la infancia. Se debe anotar que ninguno de los estudios buscó secuelas a largo plazo ${ }^{(4)}$. La pregunta pertinente sería: ¿le produciría algún daño al recién nacido negarle el 25\% o más de su volemia? Hasta la fecha apenas se ha publicado un estudio experimental en un modelo animal, que sugiere efectos adversos de la pérdida de sangre al nacimiento, en el cual los autores removieron aproximadamente el 25\% del volumen sanguíneo en ratones inmediatamente después del nacimiento. Se detectó la presencia de citocinas pro-inflamatorias en los pulmones e hígado a las tres horas de edad en los ratones a los que se les había removido sangre, en contraste con los ratones sin pérdida de sangre. ${ }^{42}$ Estos resultados avalan la importancia de reevaluar los efectos del pinzamiento inmediato del cordón umbilical. Las citocinas pro-inflamatorias son marcadores importantes de daño tisular y se 
han encontrado niveles significativamente superiores en los recién nacidos humanos que desarrollan más tarde parálisis cerebral. En consecuencia, dichas citocinas pueden ser marcadores importantes para medir cuando se estudia el efecto de diferentes prácticas obstétricas en el resultado neonatal.

\section{PINZAMIENTO TARDÍO DEL COR- DÓN UMBILICAL EN EL MANEJO ACTIVO DEL ALUMBRAMIENTO}

La hemorragia materna es una de las causas principales de mortalidad materna, especialmente en países en desarrollo, en donde se calcula que desencadena al menos el $25 \%$ de dichas muertes. Se ha demostrado que el manejo activo del alumbramiento es mejor que el manejo expectante, dado que reduce pérdida de sangre, hemorragias postparto, anemia postparto y los requerimientos de transfusiones durante el puerperio. ${ }^{43}$ El manejo activo del alumbramiento incluye la administración profiláctica de oxitocina durante o inmediatamente después del nacimiento del bebé y, por lo general, el pinzamiento temprano del cordón umbilical y tracción controlada para facilitar el nacimiento de la placenta. Sería de gran importancia utilizar la técnica que produzca más beneficios y disminuya los efectos adversos a la madre y al recién nacido. Hasta la fecha no se han evaluado los resultados maternos y neonatales del manejo activo del alumbramiento con pinzamiento tardío del cordón. Por el momento, es un protocolo de investigación propuesto por el Centro Latinoamericano de Perinatología y Desarrollo Humano (CLAP/OPS). ${ }^{44}$

\section{SITUACIONES EN LAS QUE PUDIE- RAN ESTAR ELEVADOS LOS RIESGOS DEL PINZAMIENTO TARDÍO DEL CORDÓN UMBILICAL}

Se han reportado dos condiciones con riesgo elevado para pinzamiento tardío del cordón umbilical: uso de anestesia general en la madre ${ }^{45} \mathrm{e}$ isoinmunización Rh. ${ }^{16}$ Dos condiciones en las que tradicionalmente se hace pinzamiento temprano del cordón son el parto gemelar, para evitar la transfusión feto-fetal, y en las madres portadoras del virus de la inmunodeficiencia humana, para evitar el contagio de los bebés, pero no encontramos estudios publicados al respecto y, por lo tanto, no podemos dar recomendaciones.

En el caso de depresión neonatal o líquido amniótico teñido de meconio espeso, se deben agotar todos los esfuerzos para hacer reanimación neonatal con el cordón umbilical intacto, permitiendo la transfusión placentaria de sangre oxigenada. La respuesta pobre a las medidas de reanimación en la sala de partos usualmente se atribuye a la depleción de volemia, y precisamente, el expansor de volumen ideal y el único con capacidad de transportar oxígeno es la sangre total. ${ }^{19}$ De manera similar, en el caso de circular del cordón al cuello, se deben efectuar todos los esfuerzos para reducirla, antes que pinzar el cordón umbilical mientras la cabeza del bebé aún se encuentra en el periné.

\section{RESPECTO AL PINZAMIENTO DEL CORDÓN UMBILICAL, ¿QUÉ DICE LA NORMA TÉCNICA PARA LA ATEN- CIÓN DEL PARTO DEL MINISTERIO DE LA PROTECCIÓN SOCIAL?}

En la tabla 3 se resumen las diferentes formas de pinzamiento del cordón umbilical recomendadas por el Ministerio de la Protección Social de Colombia, en su resolución 0412 del año 2000. ${ }^{47}$

\section{COMENTARIO FINAL}

No hay evidencia científica para justificar el pinzamiento temprano del cordón umbilical y cada vez hay más evidencia de los beneficios del pinzamiento tardío y de la ausencia de efectos adversos. El temor a policitemia, hiperviscosidad, hiperbilirrubinemia y taquipnea transitoria es infundado, originado de estudios meramente observacionales. Mientras no exista evidencia apropiada y suficiente que demuestre lo contrario, es mejor respetar la naturaleza que 


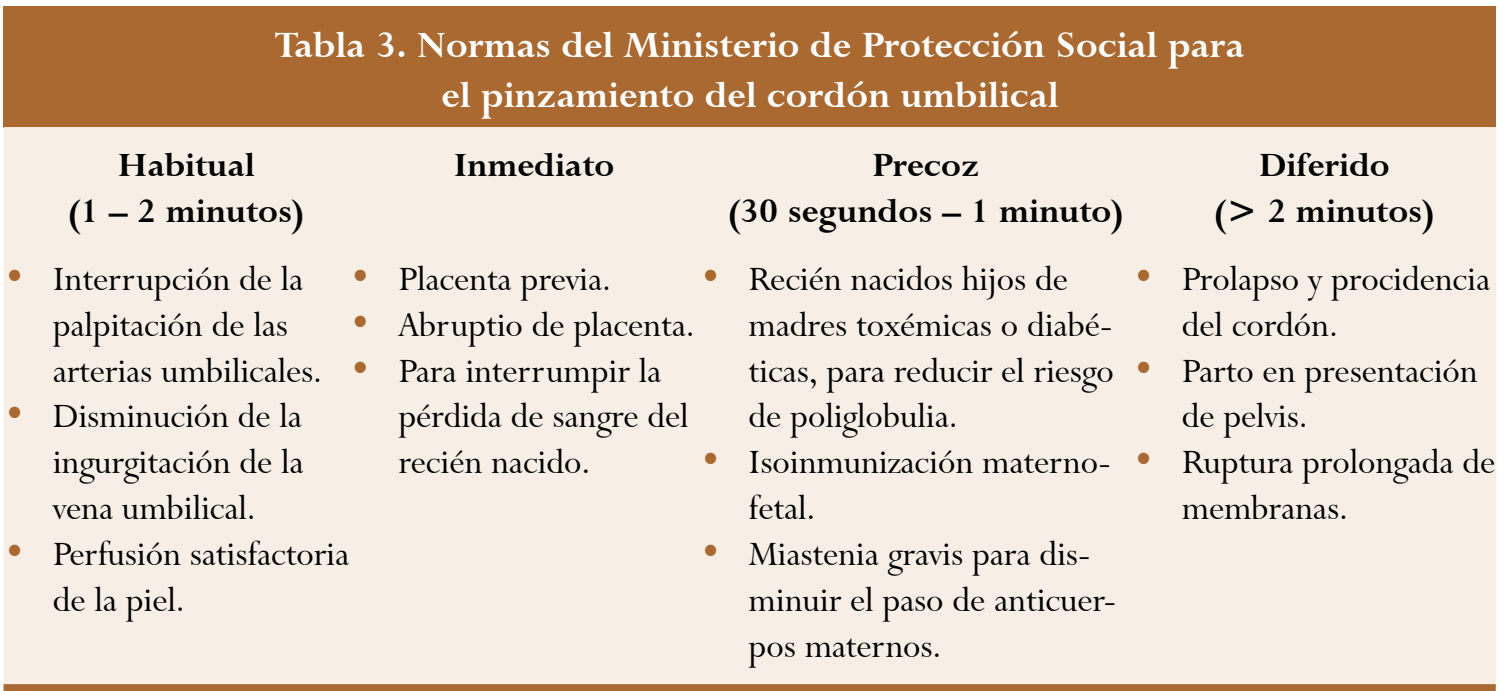

interferir con la fisiología compleja y parcialmente comprendida de la transición neonatal, como bien lo expresó el doctor Erasmus Darwin, abuelo de Charles Darwin, en al año de 1792 en su libro "Zoonomia": ${ }^{48}$ Otra cosa muy lesiva para el niño es pinzar y cortar el cordón umbilical muy pronto, el cual debe dejarse intacto no solamente hasta que el niño haya respirado repetidamente, sino hasta que las pulsaciones cesen. De manera contraria, el niño será más débil de lo que debería ser y se dejaría en la placenta una parte de sangre que debería estar en el niño; al mismo tiempo no se colapsaría naturalmente la placenta y no sería removida del útero con tanta seguridad y certeza. La Organización Mundial de la Salud considera el pinzamiento temprano del cordón umbilical una intervención y como tal requiere de justificación. ${ }^{1}$

\section{REFERENCIAS}

1. World Health Organization. Care of the umbilical cord: a review of the evidence. 1998 - WHO/RHTMSM/98.4.

2. McCausland AM, Holmes F, Schumann WR. Management of cord and placental blood and its effect upon newborn. Part II. West J Surg 1950;58:591-608.

3. Mercer JS, Nelson CC, Skovgaard RL. Umbilical cord clamping: beliefs and practices of American nurse-midwives. J Midwifery Womens Health 2000;45:58-66.

4. Mercer JS. Current best evidence: a review of the literature on umbilical cord clamping. J Midwifery Womens Health 2001;46:402-14
5. Cunningham FG. Conducta en el parto normal. En: Cunningham FG, MacDonald PC, Gant NF, Leveno KJ, Gilstrap LC (eds). Obstetricia de Williams. Cuarta edición. Barcelona: Masson SA; 1996, p. 359-80.

6. Schwarcz RL. El parto normal. En: Schwartcz RL, Duverges CA, Díaz AG, Fescina RH (eds). Obstetricia. Cuarta edición. Buenos Aires: Editorial El Ateneo; 1986. p. 310-405.

7. Júbiz AH. Atención del parto normal. En: Botero JU, Júbiz AH, Henao G (eds). Obstetricia y Ginecología. Texto Integrado. Medellín: Universidad de Antioquia; 1999. p. 114-44.

8. Taylor ES. Asistencia del parto. En: Taylor ES (ed). Obstetricia de Beck. México DF: Interamericana; 1973. p. 186-96.

9. American Academy of Pediatrics, American College of Obstetricians and Gynecologists, March of Dimes Birth Defects Foundation. Guidelines for perinatal care. Fourth edition. 1997.

10. Society of Obstetricians and Gynaecologists of Canada. Attendance at labour and delivery guidelines for obstetrical care. SOGC Policy Statement. 2000, Nº 89.

11. Waugh J, Johnson A, Farkas A. Analysis of cord blood gas at delivery: questionnaire study of practice in the United Kingdom. BMJ 2001;323:727.

12. Sociedad Colombiana de Obstetricia y Ginecología. http://www.scog.org.co

13. Sociedad Española de Ginecología y Obstetricia. http:// www.sego.es/index.php

14. Morley GM. Cord closure: can hasty clamping injure the newborn? OBG Management 1998;7:29-36. 
15. Yao AC, Moinian M, Lind J. Distribution of blood between infant and placenta after birth. Lancet 1969;7626:871-3.

16. Maternal Health and Safe Programme. Care in normal birth: a practical guide. Report of the technical working group. Geneva. Maternal and Newborn Health/Safe Motherhood Unit, Family and Reproductive Health, World Health Organization. 1997 - WHO/FRH/ MSM/96.24.

17. Yao AC, Lind J. Effect of gravity on placental transfusion. Lancet 1969;2:505-8.

18. Serwer GA. Postnatal circulatory adjustments. In Polin RA, Fox WW (eds). Fetal and Neonatal Physiology. Philadelphia: W.B. Saunders; 1992. p. 710-21.

19. Mercer JS, Skovgaard RL. Neonatal transitional physiology: a new paradigm. J Perinat Neonatal Nurs 2002;15:56-75.

20. Jaykka S. Capillary erection and lung expansion; an experimental study of the effect of liquid pressure applied to the capilary network of excised fetal lungs. Acta Paediatr 1957;Suppl 112:1-91.

21. Jaykaa S. Capillary erection and the structural appearance of fetal and neonatal lungs. Acta Paediatr 1958;47:484500 .

22. Avery ME, Frank R, Gribetz I. The inflationary force produced by pulmonary vascular distention in excised lungs; the possible relation of this force to that needed ton inflate the lungs at birth. J Clin Invest 1959;38:45662.

23. Philip AG, Saigal S. When should we clamp the umbilical cord? Neoreviews 2004;5:142-54.

24. Saigal S, U sher R. Symptomatic neonatal plethora. Biol Neonate 1977;32:62-72.

25. Bada HS, Korones SB, Pourcyrous M, Wong SP, Wilson WM 3rd, Kolni HW, et al. Asymptomatic syndrome of polycythemic hyperviscosity: effect of partial plasma exchange transfusion. J Pediatr 1992;120:579-85.

26. Saigal S, O’ Neill A, Surainder Y, Chua LB, Usher R. Placental transfusion and hyperbilirrubinemia in the premature. Pediatrics 1972;49:406-19.

27. Grajeda R, Perez-Escamilla R, Dewey KG. Delayed clamping of the umbilical cord improves hematologic status of Guatemalan infants at 2 months of age. Am Clin Nutr 1997;65:425-31.

28. Geethanath RM, Ramji S, Thirupuram S, Rao YN. Effect of timing of cord clamping on the iron status of infants at 3 months. Indian Pediatr 1997;34:103-6.
29. Rabe H, Wacker A, Hülskamp G, Hornig-Franz I, Schulze-Everding A, Harms E, et al. A randomized controlled trial of delayed cord clamping in very low birth weight preterm infants. Eur J Pediatr 2000;159:775-7.

30. McDonnell M, Henderson-Smart DJ. Delayed umbilical cord clamping in preterm infants: a feasibility study. J Paediatr Child Health 1997;33:308-10.

31. Ibrahim HM, Krouskop RW, Lewis DF, Dhanireddy R. Placental transfusion: umbilical cord clamping and preterm infants. J Perinatol 2000;20:351-4.

32. Pisacane A. Neonatal prevention of iron deficiency. BMJ 1996;312:136-7.

33. Nelle M, Fischer S, Conze S, Bedgen B, Grischke EM, Linderkamp O. Effects of later cord clamping on circulation in prematures (VLBWI) [abstract]. Pediatr Res 1998;44:454.

34. Nelle M, Zilow EP, Bastert G, Linderkamp O. Effect of Leboyer childbirth on cardiac outpout, cerebral and gastrointestinal blood flow velocities in full term neonates. Am J Perinatol 1995;12:212-6.

35. Wardrop CA, Holland BM. The roles and vital importance of placental blood to the newborn infant. J Perinat Med 1995;23:139-43.

36. Rabe H, Wacker A, Hulskamp G, et al. Late cord clamping benefits extrauterine adaptation [abstract]. Pediatr Res 1998;44:454.

37. Pietra GG, D'Amodio MD, Leventhal MM, Oh W, Braudo JL. Electron microscopy of cutaneous capillaries of newborn infants: effects of placental transfusion. Pediatrics 1968;42:678-83.

38. Oh W, Lind J. Body temperature of the newborn infant in relation to placental transfusion. Acta Paediatr Scand 1967;Suppl 172:135+.

39. Oh W, Oh MA, Lind J. Renal function and blood volume in newborn infant related to placental transfusion. Acta Paediatr Scand 1966;55:197-210.

40. Perez-Escamilla R, Pollit E, Lonnerrdal B, Dewey KG. Infant feeding policies in maternity wards and their effect on breast-feeding success: an overview. Am J Public Health 1994;84:89-97.

41. Oxford Midwives Research Group. A study of the relationship between the delivery to cord clamping interval and the time of cord separation. Midwifery 1991;7:167-76.

42. Rajnik M, Salkowski C, Li Y, et al. Early cytokine expression induced by hemorrhagic shock in a nonrescuscitated rat model. Pediatr Res 2001;49:44A. 
43. Prendiville WJ, Elbourne D, McDonald S. Active versus expectant management in the third stage of labour (Cochrane Review). In: The Cochrane Library, Issue 4, 2002. Oxford: Update Software.

44. Latin American Center of Perinatology and Human Development. Clamping modality of the umbilical cord during active management of third stage of labor: consequences to the newborn and the mother. http://www. paho.org/English/CLAP/invpro16.htm
45. Erkkola R. Kero P, Kanto J, Korvenranta H, Nanto V, Peltonen T. Delayed cord clamping in cesarean section with general anesthesia. Am J Perinatol 1984;2:165-9.

46. Ministerio de Salud de Colombia. Norma técnica para la atención del parto. 2000.

47.Dunn PM. Dr Erasmus Darwin (1731-1802) of Lichfield and placental respiration. Arch Dis Child Fetal Neonatal Ed 2003;88:346-48. 